\title{
Quand les muons cosmiques explorent le cœur de la matière
}

Sébastien Procureur (sebastien.procureur@cea.fr)

Département de physique nucléaire, Irfu, CEA - Université Paris Saclay, 91191 Gif-sur-Yvette Cedex

\section{Principe de la muographie}

Il existe aujourd'hui de nombreuses sources, pour la plupart artificielles, de rayonnements et de particules utilisables en imagerie. Selon leur nature, elles peuvent sonder différents types d'objets ou de matériaux, généralement jusqu'à des profondeurs de quelques centimètres voire dizaines de centimètres. Les structures plus profondes nécessitent des énergies ou des pénétrabilités hors d'atteinte des sources artificielles. Pour de tels cas, que l'on imagine très nombreux, la nature a mis à notre disposition une source continue et disponible partout sur Terre : les muons.

Ces particules sont issues du rayonnement cosmique primaire [1], terme impropre et qui désigne un flux de particules chargées - essentiellement des protons et des particules alpha - provenant de diverses sources dans l'Univers. L'énergie cinétique de ces particules primaires est phénoménale, et peut atteindre celle d'une balle de tennis lancée à $150 \mathrm{~km} / \mathrm{h}$, soit plusieurs millions de fois les énergies accessibles au grand collisionneur de hadrons du CERN. En arrivant au voisinage de la Terre, ces particules entrent en collision avec les atomes de la haute atmosphère et produisent une myriade d'autres particules : pions, kaons, électrons, photons... La plupart de ces dernières se désintègrent en vol ou sont absorbées dans l'atmosphère, qui agit ainsi comme un bouclier protecteur. Quelques particules parviennent cependant à franchir ce bouclier et à arriver jusqu'au sol. Il s'agit en grande majorité de muons, cousins instables des électrons - de la famille des leptons - et 207 fois plus massifs. Grâce à leur masse plus élevée, leur perte d'énergie par rayonnement - appelée Bremmstrahlung - est bien plus faible, les rendant beaucoup plus pénétrants que les électrons et donc aptes à sonder de grandes quantités de matière.

Le flux de muons au niveau du sol est relativement modeste, de l'ordre de 150 par mètre carré et par seconde, mais se caractérise par une très grande dispersion en énergie, jusqu'au-delà du $\mathrm{TeV}$ $\left(10^{12} \mathrm{eV}\right)$. Comme cette énergie détermine directement le pouvoir de pénétration des muons dans la matière, on comprend aisément qu'ils permettent de sonder des objets de tailles très diverses, de quelques centimètres à plusieurs centaines de mètres.

En pratique l'imagerie par muons, ou muographie, peut se réaliser de différentes manières, généralement déterminées par le type et la taille de l'objet à étudier [2]. - Pour des objets de petite taille (typiquement jusqu'aux dimensions d'un conteneur ou d'un camion), on utilise la muographie par déviation (fig. 1a) : des détecteurs de particules sont placés de part et d'autre de l'objet, et mesurent la direction des muons en amont et en aval. La distribution des points d'impact et des angles de diffusion permet alors d'accéder à la densité en 3D de l'objet. Les dimensions limites des objets sont déterminées par la surface de détection à instrumenter - en général quelques $\mathrm{m}^{2}$ - ainsi que par le floutage de l'image qui apparait à grande opacité(a) à cause de diffusions multiples.

- Pour les objets plus grands ou plus épais (volcans [3], pyramides, bâtiments...), on privilégie la muographie par transmission (fig. 1b) : les détecteurs sont placés uniquement d'un côté de l'objet (en contrebas), et enregistrent le 


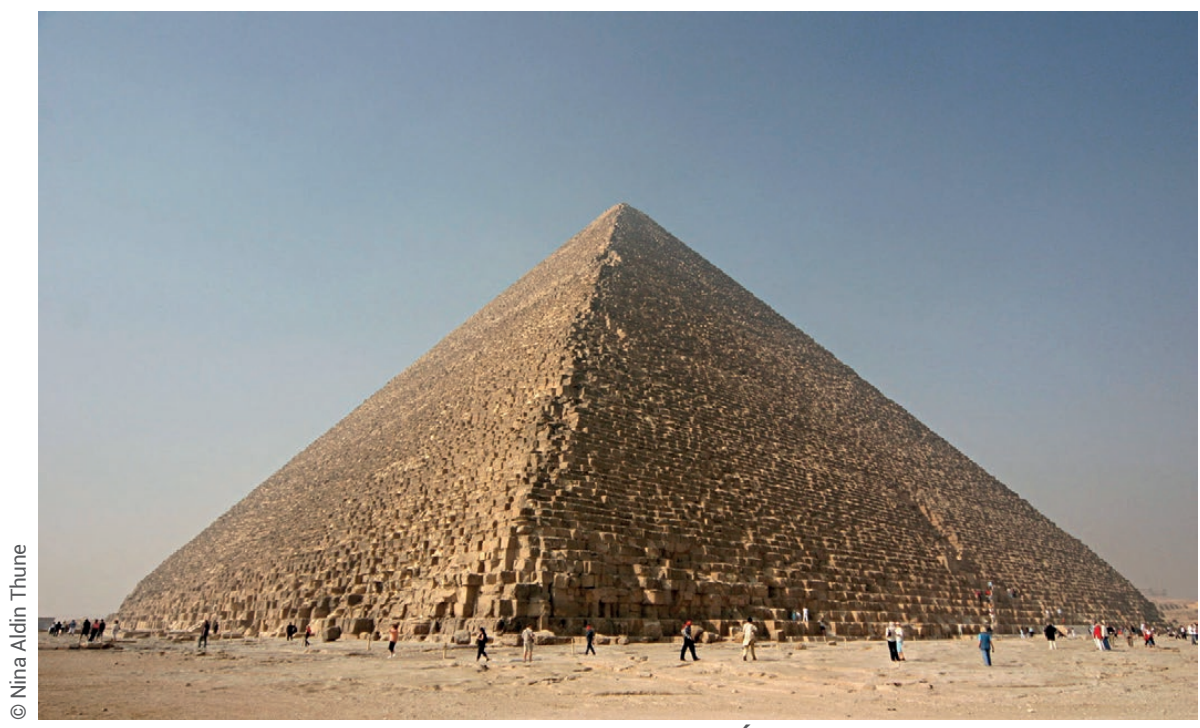

La grande pyramide de Khéops, sur le plateau de Gizeh en Égypte.

a

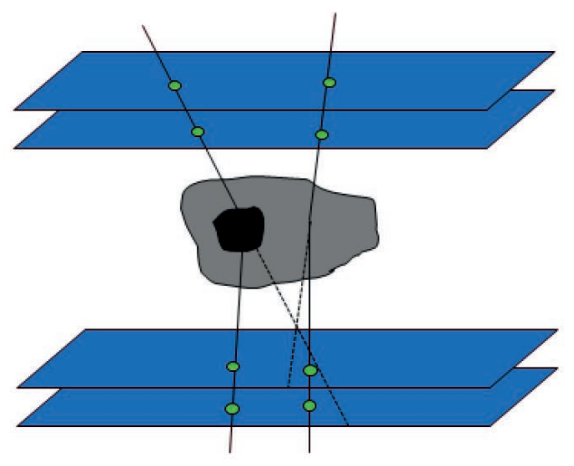

1. Dispositifs typiques de muographie en déviation (a) et en transmission (b) d'un objet (en gris), avec des détecteurs de particules (en bleu). Dans le premier cas, la reconstruction des trajectoires en amont et en aval permet de localiser des points de diffusion et leur densité via la déviation angulaire, et ainsi de former une image 3D de la densité de l'objet. Dans le second cas, le flux de muons mesuré dans les différentes directions renseigne sur l'opacité de l'objet, et donc sur sa distribution $2 \mathrm{D}$ en densité.

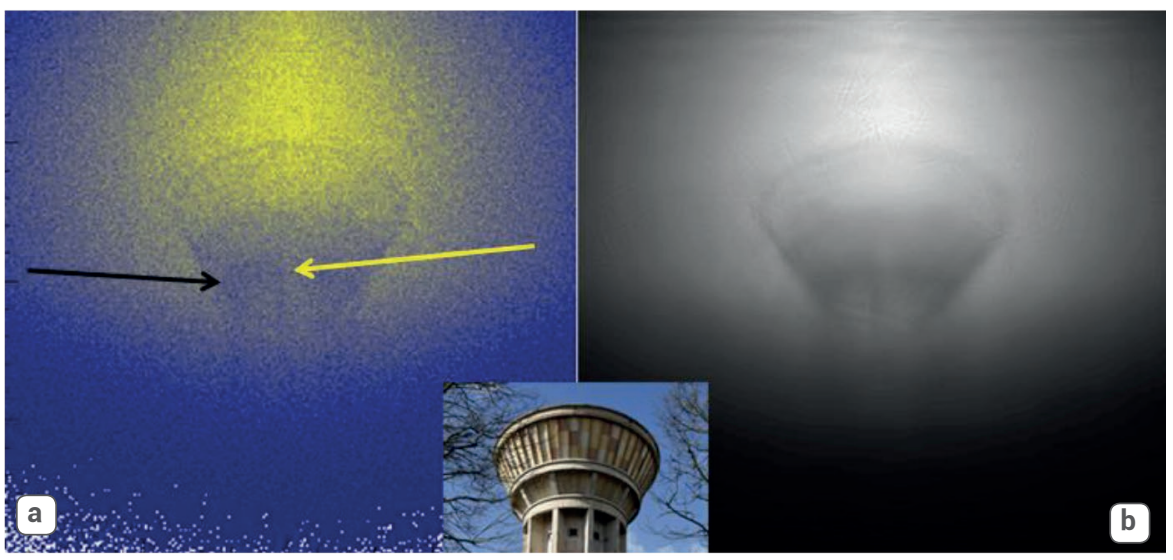

2. Muographie du château d'eau du CEA-Saclay.

(a) Muographie brute, obtenue en quatre semaines avec un télescope de $25 \mathrm{~m}^{2}$ [4].

(b) Muographie après traitement d'image et lissage (par J. Bobin).

On distingue plusieurs détails architecturaux, ainsi que la réserve d'eau (flèche noire) dans la partie inférieure de la cuve, et le vide de l'escalier en colimaçon (flèche jaune) au centre de la cuve. Cette image est à notre connaissance la première muographie en haute définition et reconnaissable d'une structure de grande dimension. flux de muons dans différentes directions. Ce flux est relié de manière directe à l'opacité de l'objet dans la direction d'observation : plus l'objet est dense ou épais, et plus le nombre de muons détectés en aval sera faible. L'information recueillie dans ce mode est moins complète qu'en déviation, et on ne peut mesurer que la distribution 2D de la densité, moyennée dans l'axe d'observation du détecteur.

- Il existe également un mode intermédiaire aux deux précédents, appelé muographie en absorption. Ce mode nécessite de placer des détecteurs de chaque côté comme en déviation, mais réalise une image bidimensionnelle comme en transmission. Dans ce cas, les détecteurs situés en dessous de l'objet identifient les muons transmis, et on reconstruit l'image seulement à partir des muons absorbés (et donc non détectés en dessous). Avec un tel dispositif, on peut en fait réaliser les trois types de muographies. L'intérêt de l'imagerie par absorption est d'offrir une alternative pour les objets d'opacité intermédiaire, i.e. trop épais pour la déviation (floutage dû aux diffusions multiples) mais encore trop minces pour la transmission.

Si les muons représentent effectivement une source naturelle, gratuite et pénétrante, il existe néanmoins des contraintes d'utilisation pratique. Le flux modeste de particules rend les temps d'acquisition assez longs, de quelques minutes ou dizaines de minutes en déviation pour 
des objets fins, jusqu'à plusieurs semaines voire plusieurs mois pour les mesures en transmission de très gros objets (fig. 2). Par ailleurs, l'absence de muons remontants nécessite de devoir toujours placer des détecteurs en contrebas, et le plus en dessous possible des objets ${ }^{(b)}$.

\section{Les instruments de la muographie}

Depuis les premiers compteurs Geiger et les chambres à brouillard du début du $\mathrm{XX}^{\mathrm{e}}$ siècle, de nombreux types de détecteurs de particules ont été mis au point pour la recherche fondamentale. Malgré tout, la muographie requiert des critères spécifiques qui ne sont pas forcément ceux de la physique des particules. En premier lieu, les instruments doivent être d'assez grande taille - typiquement plusieurs dizaines de centimètres au moins - pour pallier le flux modeste de muons. Ils doivent également être précis, pour extraire le maximum d'information de chaque muon enregistré. Pour les utilisations en extérieur, leur consommation électrique peut aussi être un élément important, ainsi que leur (trans)portabilité et leur robustesse aux chocs ou aux conditions environnementales. Réunir tous ces critères simultanément n'est pas chose aisée, surtout si on ajoute le critère (universel celui-là) du prix de revient.

Il existe ainsi aujourd'hui essentiellement trois technologies de détection utilisées en muographie, dont les caractéristiques sont résumées dans le tableau 1.

- Les émulsions nucléaires, à base d'halogénures d'argent, dont le principe est très similaire à la photographie argentique. Ces détecteurs ont une excellente résolution spatiale et ne nécessitent pas d'alimentation électrique. Ils sont en revanche sensibles à l'environnement extérieur (dégradation des films selon la température et l'humidité) et requièrent une longue analyse $a$ posteriori, les empêchant d'obtenir des images en temps réel ou en dynamique.

- Les scintillateurs plastiques, dans lesquels les muons produisent de la lumière convertie en signal électrique. Ces détecteurs sont particulièrement

\begin{tabular}{|c|c|c|c|}
\hline & $\begin{array}{l}\text { Émulsions } \\
\text { nucléaires }\end{array}$ & Scintillateurs & $\begin{array}{l}\text { Détecteurs } \\
\text { gazeux }\end{array}$ \\
\hline $\begin{array}{c}\text { Précision } \\
\text { (résolution spatiale) }\end{array}$ & quelques $\mu \mathrm{m}$ & 3 à 20 mm & 0,2 à 0,6 mm \\
\hline $\begin{array}{l}\text { Compacité } \\
\text { de l'instrument }\end{array}$ & $\begin{array}{c}\text { excellente } \\
\text { (quelques cm) }\end{array}$ & $\begin{array}{l}\text { médiocre } \\
(\sim 2 \mathrm{~m})\end{array}$ & $\begin{array}{l}\text { bonne } \\
(\sim 50 \mathrm{~cm})\end{array}$ \\
\hline Robustesse & fragile & robuste & robuste \\
\hline $\begin{array}{l}\text { Consommation } \\
\text { électrique }\end{array}$ & nulle & $\begin{array}{c}\text { quelques } \\
\text { dizaines de W }\end{array}$ & $\begin{array}{c}\text { quelques } \\
\text { dizaines de W }\end{array}$ \\
\hline $\begin{array}{l}\text { Sensibilité } \\
\text { aux conditions } \\
\text { extérieures }\end{array}$ & $\begin{array}{c}\text { sensible } \\
\text { aux températures } \\
\text { supérieures à } 25^{\circ} \mathrm{C} \\
\text { et à l'humidité }\end{array}$ & insensible & très peu sensible \\
\hline $\begin{array}{c}\text { Temps } \\
\text { d'exposition } \\
\text { maximal }\end{array}$ & $\sim 3$ mois & infini & infini \\
\hline $\begin{array}{l}\text { Obtention } \\
\text { des images }\end{array}$ & $\begin{array}{l}\text { plusieurs mois } \\
\text { (développement, } \\
\text { analyse) }\end{array}$ & temps réel & temps réel \\
\hline $\begin{array}{l}\text { Imagerie } \\
\text { dynamique }\end{array}$ & non & oui & oui \\
\hline
\end{tabular}

Tableau 1. Caractéristiques des trois différents types de détecteurs utilisés en muographie. robustes, insensibles aux conditions environnementales, et permettent une imagerie en temps réel. Leur résolution spatiale est en revanche plutôt médiocre, limitant la compacité des instruments et la qualité des images obtenues.

- Les détecteurs gazeux, généralement à base d'argon, dans lesquels les muons produisent un signal électrique par ionisation. À l'instar des scintillateurs, ils sont robustes et fonctionnent en temps réel, mais disposent d'une résolution 10 à 50 fois meilleure. Historiquement, cette technologie est la plus récente, et offre à l'heure actuelle un excellent compromis en combinant les principaux avantages des deux précédentes.

\section{Muographie de pyramides}

La possibilité de sonder la structure interne de très grands objets a suscité un intérêt pour cette technique dès le milieu $\mathrm{du} \mathrm{XX}^{\mathrm{e}}$ siècle. En effet, la présence de cavités sur le trajet des muons se traduit par un excédent du nombre de muons transmis à travers l'objet.

Dans les années 1960, le physicien américain Luis Alvarez entreprend de sonder la pyramide de Khéphren à Gizeh. Deuxième plus grande pyramide après sa voisine de Khéops, la pyramide de Khéphren aiguise particulièrement l'intérêt d'Alvarez et des archéologues, puisqu'on ne connait d'elle qu'une seule chambre, dite de Belzoni, située au centre de sa base. La configuration est donc idéale pour un instrument de muographie, et le potentiel de découverte réel. Malgré un appareillage encombrant et encore rudimentaire, et malgré les troubles du moment ${ }^{(c)}$, l'équipe du professeur Alvarez recueille plusieurs mois de données et parvient à reconstruire la forme globale de la pyramide. Mais aucune structure inconnue n'apparait sur les images. L'Histoire retiendra, un peu ingrate, qu'Alvarez n'a rien trouvé, alors qu'Alvarez explique avoir trouvé qu'il n'y avait rien...

Dans les années 2000, à l'heure où la muographie se réveille après trente ans de quasi-hibernation, un autre projet voit le jour au Mexique, pour sonder la pyramide du Soleil sur le site de Teotihuacan. Lorsqu'il apprend l'existence d'un tunnel menant au centre de cette pyramide, le professeur Arturo Menchaca entreprend la réalisation d'un télescope à 


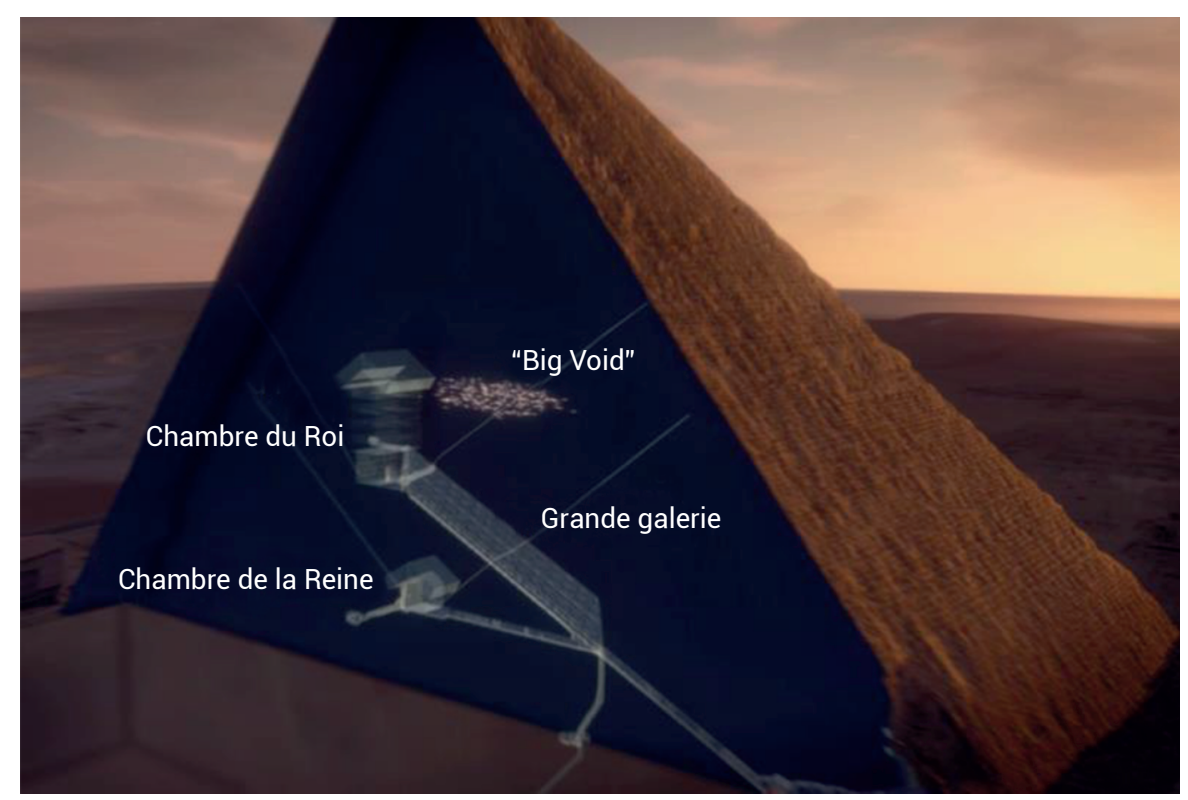

3. Coupe Nord-Sud de la pyramide de Khéops, montrant les grandes structures connues. Le "ScanPyramids Big Void", situé au-dessus de la Grande Galerie, mesure environ 30 mètres de long.

muons à base de détecteurs gazeux (chambres à fils). L'adaptation de l'instrument au terrain difficile et la campagne de mesures s'étalent sur près d'une décennie, et les données sont comparées au fur et à mesure avec des simulations du flux de muons attendu. La correspondance est excellente, et permet de déceler une légère sous densité de plusieurs dizaines de mètres dans l'édifice. Si les médias s'emparent aussitôt de ces résultats en annonçant que la pyramide risque l'effondrement tel un château de sable, l'analyse des données et leur interprétation fine en termes d'architecture interne sont toujours en cours.
En 2015, une nouvelle grande mission est lancée en Égypte. Baptisée ScanPyramids [5], elle réunit des scientifiques égyptiens, japonais, français et canadiens dans le but de sonder les quatre plus grandes pyramides de la $I^{\mathrm{e}}$ dynastie : les pyramides rouge et rhomboïdale à Dahchour, et les pyramides de Khéphren et Khéops à Gizeh. Conçue et coordonnée par Mehdi Tayoubi (Institut HIP [6]) et le professeur Hany Helal (Université du Caire) sous l'autorité du Ministère des Antiquités égyptiennes, ScanPyramids propose d'étudier ces édifices à l'aide des dernières techniques développées en muographie, mais aussi en imagerie thermique et aérienne (drones). Côté muographie, dès 2013 deux équipes japonaises de l'Université de Nagoya (K. Morishima) et du centre japonais KEK de recherches sur les particules (F. Takasaki) sont contactées. Après l'annonce du lancement de la mission en octobre 2015, le CEA propose sa participation et rejoint officiellement la mission en avril 2016. Pour la première fois, les trois technologies majeures de la muographie se retrouvent sur un même site et une même collaboration.

Après une première campagne de mesures dans la pyramide rhomboïdale, les émulsions de Nagoya sont installées dès le début 2016 dans le couloir descendant et la Chambre de la Reine de la pyramide de Khéops (fig. 3). Le grand télescope à scintillateur du KEK, également prévu pour la Chambre de la Reine, commence les prises de données à l'été. Côté CEA, trois télescopes à base de Micromegas - un détecteur gazeux à micro-pistes - se déploient en extérieur autour de la pyramide au mois de juin. Ces choix d'emplacements sont techniques, mais aussi stratégiques. Techniquement, les émulsions sont trop fragiles et le scintillateur trop peu précis pour être installés à l'extérieur. Les télescopes du CEA, précis et robustes, ont encore besoin d'un renouvellement de gaz en continu pour compenser les fuites résiduelles et le dégazage des différents matériaux. Stratégiquement, ce déploiement permet aussi de sonder des zones complémentaires de la pyramide.

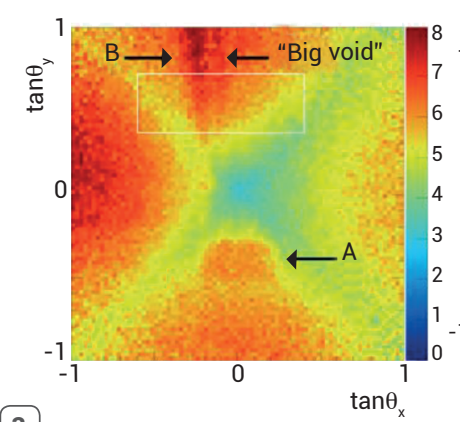

a

4. Muographies obtenues par l'Université de Nagoya (a), le KEK (b) et le CEA (c), démontrant l'existence du "ScanPyramids Big Void". Un nombre de muons élevé se traduit par une couleur plus rouge (a et c) ou blanche (b). Sur l'image (a), la Chambre du Roi (A) et la Grande Galerie (B) sont également visibles. L'image (b) est obtenue en soustrayant le flux de muons attendu, ce qui laisse apparaitre uniquement les structures inconnues (le "Big Void" apparaît comme une ligne blanche verticale). Sur l'image (c), les rectangles blancs indiquent les excédents de muons des deux histogrammes de droite. Ces deux excédents correspondent à la Grande Galerie (en bas) et au "Big Void" (en haut). 


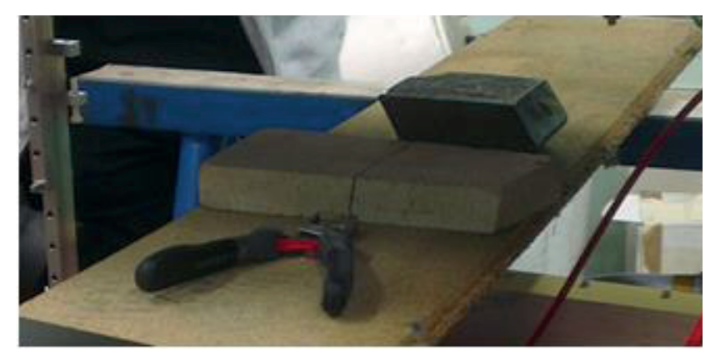

a

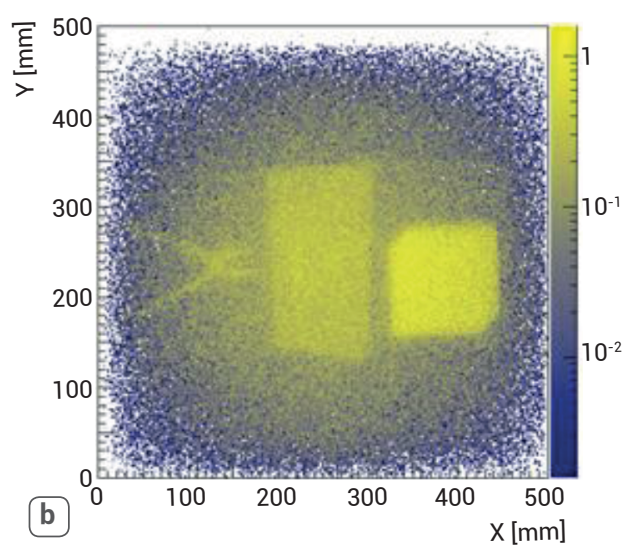

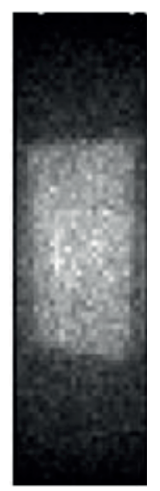

c

5. Photographie (a) et muographie en déviation (b-c) de petits objets : une pince coupante, une pierre calcaire et une brique de plomb, posés sur une planche en bois. La précision de la muographie permet même de visualiser le rebord de la planche, malgré les très faibles écarts de densité. Un zoom sur la pierre calcaire (c) permet aussi de repérer l'armature métallique interne (ligne verticale claire à la gauche de l'image).

$>>$

Fin 2016, après plusieurs mois de prises de données, deux premières découvertes sont annoncées : les émulsions de Nagoya situées dans le couloir descendant révèlent l'existence d'un vide allongé partant de la zone des Chevrons sur la face Nord. Les télescopes du CEA observent quant à eux une petite cavité à environ 110 mètres de hauteur près de l'arête Nord-Est, en plus d'une cavité similaire déjà connue 30 mètres plus bas. Dans les deux cas, l'analyse des muographies montre par endroits des excédents de muons significatifs, révélant la présence de sous-densités. L'amplitude des excédents observés, la comparaison à des vides existants, leur localisation précise depuis plusieurs points de vue, les outils de simulation ainsi que les quantités de matière présentes dans les directions correspondantes permettent de conclure de manière non ambigüe à la présence de vides.

Début 2017, les premiers résultats des émulsions placées dans la Chambre de la Reine révèlent un troisième excédent de muons significatif, cette fois-ci au voisinage de la Grande Galerie. Les données du KEK et du CEA dans cette zone sont alors analysées et montrent des excédents de muons compatibles géographiquement. Dans les mois qui suivent, une campagne de mesures dédiée est organisée, en ajoutant de nouvelles émulsions dans la Chambre de la Reine, en déplaçant le scintillateur, et en redéployant deux télescopes du CEA devant la face Nord. Une à une, les trois technologies observent l'excédent situé au-dessus de la Grande Galerie (fig. 4) [7]. Les directions précises obtenues à partir des émulsions et des Micromegas pointent exactement dans la même zone, ce qui permet d'affiner la position de ce vide par triangulation. De par leur faible distance à ce vide baptisé "ScanPyramids Big Void" et leur grande surface, les émulsions obtiennent l'image la plus précise et fournissent une estimation de son volume, qui est de plusieurs centaines de mètres cubes. Malgré leur grande distance et leur angle d'observation très incliné, les télescopes du CEA détectent également la Grande Galerie, réalisant au passage la première détection depuis l'extérieur de vides aussi internes d'une pyramide.

\section{Muographie de petits objets}

Grâce à son mode en déviation, la muographie peut aussi sonder des objets beaucoup plus petits, et renseigner ainsi sur leur distribution de matière. Cette variante requiert des détecteurs d'excellente résolution spatiale, afin de mesurer des déviations angulaires de l'ordre du milliradian. La figure 5 montre la muographie 2D de plusieurs petits objets, obtenue en une journée environ avec les détecteurs Micromegas du CEA. L'un de ces objets est un échantillon de pierre calcaire fournie par le LRMH (Laboratoire de Recherche sur les Monuments Historiques) et contenant une petite armature métallique interne. Un zoom sur cette image (fig. $5 c$ ) révèle en effet la localisation et la longueur de la tige.

La possibilité de réaliser des images sans amener de source et de manière non destructive ouvre évidemment des champs d'applications pour l'étude des objets ou matériaux anciens. Ces mesures peuvent ainsi aider à l'identification de l'objet ou de son époque - par exemple par la forme des armatures internes - ou déterminer et guider d'éventuels travaux de restauration - fragilité d'une zone à cause d'une cavité ou d'un défaut interne. D'autres mesures ont d'ailleurs récemment été menées sur des objets plus épais avec les mêmes détecteurs, et ont montré que des cavités de quelques centimètres pouvaient être localisées dans des bétons de $50 \mathrm{~cm}$ d'épaisseur.

(a) L'opacité dans une direction d'observation est définie comme la densité de l'objet intégrée sur son épaisseur.

(b) En effet, le flux de muons est maximal à la verticale et s'atténue globalement en cosinus carré de l'angle zénithal.

(c) La guerre des Six Jours a éclaté le lendemain même du début de la prise de données. L'Égypte suspectant les États-Unis d'apporter leur aide logistique à Israël, l'équipe d'Alvarez est contrainte de se retirer jusqu'à la fin du conflit, dans un climat que l'on imagine tendu.

\section{Références}

$1 \cdot \mathrm{K}$. Kotera, Reflets de la physique 32 (2013) 8-13.

2. S. Procureur, Nucl. Instr. \& Meth. A878 (2018) 169.

3. D. Gibert et al., Reflets de la physique 32 (2013) 14-18.

$4 \cdot$ S. Bouteille et al., Nucl. Instr. \& Meth. A834 (2016) 223.

$5 \cdot$ www. scanpyramids. org/?lang=fr .

6- Heritage Innovation Preservation Institute, www.hip.institute/ .

7• K. Morishima et al., Nature 552 (2017) 386. 